\title{
Successful bone reconstruction after bortezomib therapy in a myeloma patient
}

\author{
Takayuki Tanaka • Ryoko Yamasaki • \\ Hiromi Omura • Norihiko Hino
}

Received: 14 July 2011/Revised: 2 August 2011/Accepted: 2 August 2011/Published online: 23 August 2011

(c) The Japanese Society of Hematology 2011

A 50-year-old Japanese woman was referred to our hospital with intractable back pain. Laboratory examination revealed that she was suffering from Bence-Jones kappa multiple myeloma (Stage III A, ISS: Stage II). Plain X-ray revealed multiple lytic lesions in her cranial bones, ribs, and ilium. The left iliac bone defect was particularly large (Fig. 1a). She was considered to be a candidate for autologous peripheral blood stem cell transplantation (PBSCT), and received vincristine, adriamycin, and dexamethasone (VAD) as an initial induction therapy. After the first course of VAD, she experienced severe bradycardia with hypotension. In addition, on treatment with zolendronic acid (ZA), she developed a high fever. Due to her adverse responses to VAD and ZA, she was administered bortezomib $\left(1.3 \mathrm{mg} / \mathrm{m}^{2}\right.$, intravenously, on days 1, 4, 8, and 11). Her serum ALP level was elevated from 2 weeks after the beginning of bortezomib treatment, but she experienced no adverse effects other than peripheral neuropathy (Grade 2 for CTCAE ver. 4). After five courses of bortezomib treatment, her CT showed new bone formation at the lytic lesion of the left iliac bone (Fig. 1b). Moreover, improvement of the bone lesion was maintained even after the cessation of eight courses of bortezomib treatment (Fig. 1c, d), and it was determined that she had achieved a very good partial response (VGPR). As she strongly desired to be followed without any therapy, PBSCT has not been performed subsequently.

Bone disease in myeloma is commonly characterized by increased bone resorption. However, in myeloma patients, there is also impaired osteoblast activity. Bortezomib is a

T. Tanaka $(\bowtie) \cdot$ R. Yamasaki $\cdot$ H. Omura $\cdot$ N. Hino Department of Internal Medicine, Hematology,

Tottori Prefectural Central Hospital, 730 Ezu,

Tottori, Tottori 680-0901, Japan

e-mail: tanaka-tak@pref.tottori.jp
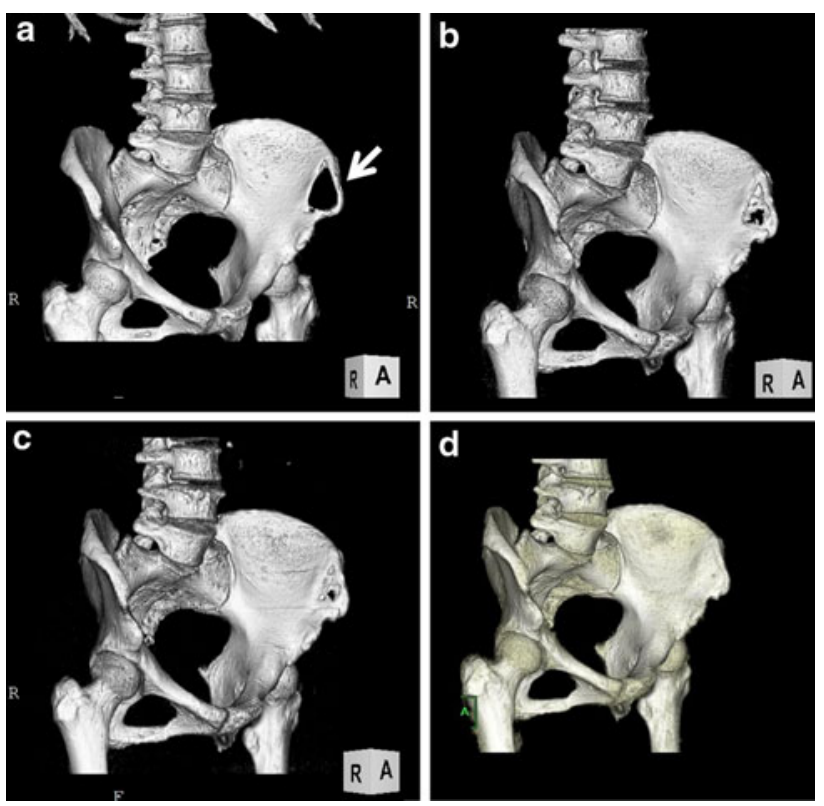

Fig. 1 Images of 3-dimensional computed tomography. a Before bortezomib therapy. A large bone defect in the left iliac bone. b After five courses of bortezomib therapy. New bone formation is demonstrated in the osteolytic lesion. c Two months after treatment. d Eighteen months after treatment. The bone defect has been completely replaced by normal bone tissue

proteasome inhibitor that has been shown to exhibit antimyeloma activity and to stimulate bone formation. In this case, we observed the radiographic improvement of a myeloma bone lesion during and following bortezomib therapy. We suggest that bortezomib may play an important role in reducing the risk of skeletal complications such as pathological fractures. 